A014 Intraoperative Lumbar Subarachnoid Drain Placement-Challenges and Complications

Liby G. Pappachan, ${ }^{1}$ Georgene Singh, ${ }^{1}$ Ramamani Mariappan, ${ }^{1}$ Mridul S. Koshy, ${ }^{1}$ Karen R. Lionel ${ }^{1}$

${ }^{1}$ Department of Anaesthesia, Christian Medical College (CMC), Vellore, Tamil Nadu, India

Background: Lumbar subarachnoid drain (LSAD) is a closed sterile catheter system placed in the lumbar subarachnoid space to drain cerebrospinal fluid (CSF) perioperatively to assist in endoscopic skull base surgeries. The placement and use of LSAD are associated with technical challenges and complications.

Materials and Methods: This prospective observational study was conducted after IRB approval. All patients planned for endoscopic TNTS/skull base tumor excision requiring LSAD were studied. LSAD was placed using 18-G epidural catheter either under GA or under LA according to the discretion of the anesthesiologist. The following parameters were noted: number of attempts, number of levels attempted for identifying the space, siting the catheter and for free flow of CSF and the volume of CSF drained. The primary investigator followed up all patients and documented complications associated with the LSAD as per defined criteria.

Results: A total of 50 patients were studied. Only in 18 patients (36\%) the tapping and siting was done at $1 \mathrm{st}$ attempt, rest 32/50 had difficulty in placing either for tapping (29/32) or siting the catheter (15/32). Fifteen patients needed $>2$ attempts and in 13/32 patients, it was tried in $\geq$ 2 levels. In 16/50 patients needed manipulation for free flow of CSF. 23/50 patients had head ache, 13/50 had backache which had correlated with number of attempts. No patient had meningitis, paresthesia, nerve root irritation, or PDPH needing epidural blood patch.

Conclusions: Though the rate of technical difficulties while placing LSAD using the epidural catheter was unacceptably high, the incidence of major complications was lower in our series.

\section{A015 A Prospective Randomized Controlled Study to Analyze the Efficacy of Scalp Block in Providing Analgesia for Supratentorial Craniotomies Using Surgical Pleth Index, Response Entropy, and Hemodynamics Manchala R. Kumar, ${ }^{1}$ Nitin Manohar, ${ }^{1}$ Astha Palan, ${ }^{1}$ Manjunath S.T. ${ }^{1}$ \\ 'Department of Neuroanaesthesia, Yashoda Hospitals, Secunderabad, Telangana, India}

Background: Supplementing general anesthesia with regional technique has markedly improved intraoperative hemodynamics and intraoperative analgesia. We compared the effects of scalp block (study group) and no scalp block (control group) on intraoperative hemodynamics and correlated surgical plethysmographic index with other variables like response entropy, state entropy and mean arterial pressure to assess the depth of analgesia.

Materials and Methods: In this prospective study, a total of 30 ASA physical grade- 1 and -2 patients, satisfying the inclusion criteria, undergoing supratentorial craniotomies were double blinded, and divided into two groups (study group, control Group).Study group received $0.2 \%$ ropivacaine $20 \mathrm{~mL}$ for scalp block and control group received $20 \mathrm{~mL}$ normal saline for scalp block and for both the groups received fentanyl infusion at $1 \mu \mathrm{g} / \mathrm{kg} / \mathrm{h}$. Intraoperatively injection fentanyl $(1 \mu \mathrm{g} / \mathrm{kg})$ was given as rescue analgesia when there was $>20 \%$ raise in hemodynamics above baseline. Quality of analgesia was assessed by using surgical plethysmographic index, response entropy and state entropy at different time points (induction, postinduction, intubation, postintubation, pin, postpin, incision, postincision, craniotomy, postcraniotomy, durotomy, and postdurotomy).

Results: We found that patients in study group had stable intraoperative hemodynamics with decrease in requirement of intraoperative fentanyl. Intraoperative fentanyl consumption (in $\mu \mathrm{g} / \mathrm{kg}$ ) was decreased in in study group $(0.06 \pm 0.04)$ compared with control group (1.73 \pm 0.52). We found that surgical plethysmographic index, response entropy, state entropy, and mean arterial pressure positively correlated intraoperatively as depth of analgesia monitors.

Conclusion: We conclude that scalp block when given along with general anesthesia can offer better intraoperative hemodynamic stability and decreased intraoperative fentanyl requirement. Surgical plethysmographic index, response entropy, and static entropy correlated well as depth of analgesia monitors.

A016 The Effect of Change of Position on Surgical Pleth Index in Patients Undergoing Lumbar Spine Surgery under General Anesthesia

Smita Musti, ${ }^{1}$ Sonia Bansal, ${ }^{2}$ Dhritiman Chakrabarti ${ }^{2}$

'Department of Anaesthesia, M S Ramaiah Medical College,

Bengaluru, Karnataka, India

${ }^{2}$ Department of Neuroanesthesiology and Neurocritical

Care, National Institute of Mental Health and Neuro Sciences (NIMHANS), Bengaluru, Karnataka, India

Background: To study the effect of change in position from supine to prone position on surgical pleth index (SPI) under GA. Correlation between SPI and heart rate (HR), mean arterial pressure (MAP), and pulse pressure variation (PPV).

Materials and Methods: After informed written consent, 25 patients undergoing lumbar spine surgery were recruited into study. Patients were connected with ECG, $\mathrm{NIBP}, \mathrm{SpO}_{2}$, temperature monitors. Induction was with fentanyl $2 \mu \mathrm{g} / \mathrm{kg}$, titrated dose of propofol, and vecuronium 0.1 $\mathrm{mg} / \mathrm{kg}$. Patient was intubated and ventilated to maintain ETCO2 32 to $35 \mathrm{~m} \mathrm{Hg}$. Morphine $0.1 \mathrm{mg} / \mathrm{kg}$ was given intravenously. Anesthesia was maintained with propofol TCI pump and fentanyl infusion $1 \mu \mathrm{g} / \mathrm{kg} / \mathrm{h}$ to achieve the static entropy of 40 to 60 . Arterial line was inserted. HR, MAP, PPV, and SPI were recorded in supine position $0,5,10,15$, and 20 minutes after positioning patient in prone at $0,5,10,15$, and 20 minutes. Additionally, they were recorded at 0, 2, and 5minutes pre- and postskin incision, muscle splitting, and laminectomy. Repeated measures data were analyzed 
within each time point block and between the time point blocks using linear mixed effect models with random intercept by the subject.

Results: There was significant increase in SPI after positioning the patient in prone position $(p \leq 0.001)$. There was no significant change in MAP and HR but PPV showed significant change when patients were positioned prone ( $p=0.001)$. Moderate correlation was noted between SPI and MAP. Negative correlation was noted between SPI and PPV.

Conclusions: SPI changes when position is changed from supine to prone. Therefore, interpretation of SPI as a surrogate measure of nociception-antinociception balance could therefore be confounded in various situations.

\section{A017 Bronchoscopy-Guided Percutaneous Dilatational Tracheostomy in Critically III Patients: A Study of Complication and Expanded Utility}

Suprith C., ${ }^{1}$ Madhusudan Kalluraya, ${ }^{1}$ Deepak Ramachandra, ${ }^{1}$ Mehta R. ${ }^{1}$

${ }^{1}$ Critical Care Medicine, Apollo Speciality Hospital, Jayanagar, Bengaluru, Karnataka, India

Background: Bronchoscopy-guided percutaneous dilatational tracheostomy (BG-PDT) is one of the most frequently performed procedures in critically ill patients. Complications occur in $5 \%$ to $40 \%$ of tracheostomies depending on study design, patient follow-up, and the definition of the different complications. The mortality rate of PCT is less than $2 \%$. We conducted this study to assess the complications and expanded utility of BG-PDT in the Indian setting.

Materials and Methods: A retrospective analysis of 116 BG-PDT performed in the past 3 years was done. PDT was done using the standard technique, using the Cook PDT kit and videobronchoscopy guidance, with all steps done under vision. Postprocedure, all clots which migrated to the distal airway were suctioned. Complications were noted. Additional BAL was done in patients with infiltrates for cultures.

Results: One hundred sixteen BG-PDT were performed. The most common indication for BG-PDT was anticipated prolonged ventilator stay to facilitate weaning. Complications were divided into intra-procedural and early postprocedural complications. Positive BAL culture was also analyzed.

Conclusions: Our study shows that BG-PDT is safe with minimal complications, when performed by experienced ICU personnel. The advantages include confirming all steps under vision, postprocedure suctioning of clots, and BAL done in the same setting. We propose BG-PDT as an "expanded PDT strategy," combining a safe visually guiding procedure with clot clearance, and additional BAL for cultures.

\section{A018 Observational Study of Intracranial Pressure Variation Using Ultrasonography Guided Optic Nerve Sheath Diameter in Trendelenburg Position among Major Abdominal Laparoscopic Surgery \\ Shobha Purohit, ${ }^{1}$ Gaurav Sharma, ${ }^{1}$ Deeksha Singh ${ }^{1}$ \\ ${ }^{1}$ Department of Anaesthesiology, SMS Medical College and \\ Hospital, Jaipur, Rajasthan, India}

Background: Laparoscopic surgeries are widely established due to its various benefits. It requires adequate surgical exposure that is achieved by pneumoperitoneum often combined with Trendelenburg position that collectively causes increase in intra-abdominal pressure leading to many systemic physiological consequences including increase in intracranial pressure. Noninvasive USG guided ONSD was used as their measurement correlates with invasive method in patients of neurocritical care unit. We aimed to investigate the effect of Trendelenburg position with pneumoperitoneum on ONSD to examine possible changes in ICP in patients undergoing major laparoscopic abdominal surgery.

Materials and Methods: Study included 40 patients of ASA I, II with no neurological disease undergoing major abdominal laparoscopic surgery. USG guided ONSD was measured in both eyes $3 \mathrm{~mm}$ behind the globe preoperatively, following induction, 3 min after Trendelenburg position and 3 min with pneumoperitoneum, 2 minutes after desufflation; other parameters observed were HR, SBP, DBP, MAP, Ppeak, and ETCO2.

Results: ONSD was significantly increased from baseline in Trendelenburg position $(p<0.001)$. Mean ONSD at Trendelenburg position $(5.2 \pm 0.8)$ was significantly more than that in supine position $(4.7 \pm 0.7)$. Four patients had ONSD > $5.8 \mathrm{~mm}$ (the cutoff value for prediction of ICP above $20 \mathrm{~mm} \mathrm{Hg}$ in previous studies). Mean ONSD at Trendelenburg with pneumoperitoneum (5.6 \pm 0.8$)$ increased compared with supine position. Seventeen patients had ONSD > $5.8 \mathrm{~mm}$.

Conclusions: We conclude that ONSD which reflects ICP was significantly increased from baseline in Trendelenburg's position with pneumoperitoneum.

\section{A019 Hydrocephalus Secondary to Cervical Spinal Cord Surgery \\ Subhajit Guha, ${ }^{1}$ Indranil Ghosh, ${ }^{1}$ Bibhukalyani Das ${ }^{1}$ \\ ${ }^{1}$ Department of Neurocritical Care, Institute of Neurosciences Kolkata (INK), Kolkata, West Bengal, India}

Background: Cerebrospinal fluid (CSF) leakage is a common complication after spinal tumor resection that resolves naturally in many cases. Hydrocephalus with CSF leakage as a complication after spinal surgery is rare. Here, we report a rare case of delayed hydrocephalus due to CSF leakage after cervical cord tumor surgery.

Case Description: A 60-year-old lady with gait disturbance and numbness of both hands underwent uneventful surgery of C1-C2 partial laminectomy and excision of schwannoma. Three months after surgery she was readmitted with features of postoperative meningitis. CSF examination was normal while CT scan showed very small pseudomeningocele. She improved with antipyretics and empirical antibiotics. Two months after this episode she presented in a deeply comatose state. CT brain revealed gross hydrocephalus and she became conscious only after VP shunt.

Conclusion: Hydrocephalus due to CSF leakage is a rare complication after spinal surgery. In our case, CSF did not prove septic meningitis. However, inflammation may 